\title{
A Microstrip Second-Iteration Square Koch Dipole Antenna for TT\&C Downlink Applications in Small Satellites
}

\author{
Jorge Simón, ${ }^{1}$ José Luis Alvarez-Flores, ${ }^{2}$ Juvenal Villanueva-Maldonado, \\ Víctor Hugo Castillo-Topete, ${ }^{3}$ Leonel Soriano-Equigua, ${ }^{3}$ and Jorge Flores-Troncoso ${ }^{4}$ \\ ${ }^{1}$ CONACYT, Centro de Investigación y Desarrollo en Telecomunicaciones Espaciales, Universidad Autónoma de Zacatecas, \\ Ramón López Velarde 801, Zacatecas, ZAC, Mexico \\ ${ }^{2}$ Coordinación General de Investigación Científica, Universidad de Colima, Gonzalo de Sandoval 444, Colima, COL, Mexico \\ ${ }^{3}$ Facultad de Ingeniería Mecánica y Eléctrica, Universidad de Colima, Carr. Colima-Coquimatlan Km 8.5, Coquimatlán, COL, Mexico \\ ${ }^{4}$ Centro de Investigación y Desarrollo en Telecomunicaciones Espaciales, Universidad Autónoma de Zacatecas, \\ Ramón López Velarde 801, Zacatecas, ZAC, Mexico \\ Correspondence should be addressed to Jorge Simón; jsimonr@gmail.com
}

Received 16 November 2016; Revised 29 January 2017; Accepted 13 February 2017; Published 9 March 2017

Academic Editor: Herve Aubert

Copyright (C) 2017 Jorge Simón et al. This is an open access article distributed under the Creative Commons Attribution License, which permits unrestricted use, distribution, and reproduction in any medium, provided the original work is properly cited.

A microstrip second-iteration square Koch dipole fractal antenna is presented. This meandered antenna has a total length of $56.56 \mathrm{~cm}$ including its feed gap and was printed on the diagonal of a $100 \mathrm{~mm} \times 100 \mathrm{~mm}$ PCB card that acts as CubeSat face. The antenna that was designed to optimize space shows acceptable performance at its resonance frequency of $455 \mathrm{MHz}$ within the 70 centimeter band, a band that is commonly used for TTC CubeSat subsystems. The designed fractal antenna shows a reflection coefficient below $-20 \mathrm{~dB}$, a VSWR below 1.2, a $-10 \mathrm{~dB}$ bandwidth of $50 \mathrm{MHz}$, and impedance magnitude of $56 \Omega$, while the average maximum gain around its resonance frequency is $2.14 \mathrm{dBi}$. All these parameters make this designed antenna suitable for small satellite applications at a band where a linear $\lambda / 2$ dipolar antenna working at $455 \mathrm{MHz}$ would be about $32.97 \mathrm{~cm}$ long, which does not fit within the largest dimension of a CubeSat face corresponding to $14.14 \mathrm{~cm}$.

\section{Introduction}

Satellites are objects or devices that orbit a planet, which can be either natural or artificial. Regarding artificial satellites, thousands of them orbit the earth and have become a very important technology for modern life [1]. Today, this space infrastructure offers telecommunication services such as television, telephony, and Internet. Satellites also allow us to predict weather, track hurricanes, monitor agricultural activities, understand population growth, and assess pollution levels [2]. With respect to classification, satellites are classified based on some specific characteristics such as their mass, which classifies them from picosatellites $(0.1-1 \mathrm{~kg})$ to large satellites $(>1000 \mathrm{Kg})$ [3].

Within the category of picosatellites are CubeSats, miniaturized satellites first developed by Bob Twiggs from Stanford University and Jordi Puig-Suari from California Polytechnic State University in 1999. CubeSats work typically in low earth orbits and have been used primarily by universities for research and space exploration, and they, as well as larger satellites, provide a wide range of research projects and applications. CubeSats are subject to a design protocol that specifies maximum outer dimensions equal to $100 \times 100 \times 100 \mathrm{~mm}^{3}$ and it is worth mentioning that their electronic equipment is made of commercial off-the-shelf components [4-6]. The main reason for miniaturizing satellites is primarily to reduce deployment costs, since larger satellites require larger rockets of greater cost to finance. Small satellites are not short of technical challenges, because they use innovative propulsion, attitude control, computer, communication, and power systems. Small satellites, as any wireless telecommunication system, also need to include antenna structures to perform core satellite functions [4]. Each of these functions requires different antenna configurations, which have commonly been helices, monopoles, and patches. CubeSats commonly work at bands for amateur satellite communication purposes such 
as $434.8-438$ (70-centimeter band), 1260-1270, and 2400$2450 \mathrm{MHz}$ bands [5-7].

Requirements as operating frequencies for CubeSats can be achieved using microstrip antennas, which are manufactured by means of standard printed-circuit-board (PCB) techniques. Many advances in terms of printed antennas for small satellites have been presented, which is evidence of their importance. An example of these advances is the one referring to planar antennas at $2.225 \mathrm{GHz}$ for small satellites in 1994 where optimization of the area between solar cells and microstrip antennas was achieved [8]. Other examples of microstrip antenna structures for small satellites are the patch antennas for the USUsat, including patch antennas for the up/downlinks resonating at $450 \mathrm{MHz}$ and $2.26 \mathrm{GHz}$, respectively [9], and the square patch low-gain microstrip antenna at $2.45 \mathrm{GHz}$ to optimize antenna placement for the ESEO and SSETIExpress [10]. Most antenna implementations target either the TTC or the payload data downlink subsystem [4] although recently a study on intersatellite cross-link antennas for picosatellites operating at $10.5 \mathrm{GHz}$ has been conducted; this study consisted of modified Van Atta retrodirective arrays [11].

Printed antennas for small satellites have advantages such as integrating them into the structure, but, in scenarios like CubeSats, a challenging task is to print them on their reduced faces $\left(100 \times 100 \mathrm{~mm}^{2}\right)$ and make them resonate at relatively large wavelengths (70-centimeter band), since conventional $\lambda / 2$ dipoles working at those frequencies are about $35 \mathrm{~cm}$ long and could not fit within those limited areas [4-6]. The aforementioned design challenge can be solved by using fractal antennas whose geometry is created through the successive iteration of a generator shape to a simple basis shape [12], using conventional antennas of size less than a quarter wavelength of the radiation bandwidth, and hence the efficiency is reduced [13]. Fractal antennas are multiband and optimize dimensions, making antennas at relatively small spaces possible work at relatively low frequencies. Fractal geometries were first studied by Mandelbrot during the 1970 s and characteristics that define them include self-similarity, where small regions of the geometry duplicate the overall geometry on a reduced scale, which is generally interpreted as a measure of the space-filling properties and complexity of the fractal shape [14-17].

In this work, a planar and low-cost antenna for TTC (telemetry, tracking, and command) subsystems of CubeSat satellites is presented. The antenna consisted of a microstrip second-iteration square Koch dipole fractal antenna working at centimeter band, which shows characteristics that make it suitable for this kind of application.

\section{Materials and Methods}

This work was based on a methodology, which considered four critical stages. The stages were (i) antenna design and configuration, (ii) simulation, (iii) antenna fabrication, and (iv) measurements and implementation. These stages are described in Sections 2.1 to 2.4.
2.1. Antenna Design and Configuration. The antenna design consisted of a microstrip fractal dipole with its 2 arms formed by square Koch curves in its second iteration $(n=2)$. The square Koch curve is obtained by repeatedly replacing each line segment composed of four quarters with the generator consisting of eight pieces, each one-quarter long. Koch curves, as other fractals, allow the optimization of the total antenna length making it possible to have antennas resonating at 70-centimeter band on a CubeSat face [18]. In this case, the Koch antenna was built on the total diagonal of a $100 \mathrm{~mm} \times 100 \mathrm{~mm}$ commercial PCB card which will act as one of the CubeSat faces. The second iteration Koch antenna occupies the entire diagonal $L_{0}=14.14 \mathrm{~cm}$ and had a total length of $L_{2}=56.56 \mathrm{~cm}$ considering both antenna arms and the feed gap, since this total dipole length of the $n$th iteration " $L_{n}$ " is given by $L_{n}=2^{n} L_{0}$, where " $n$ " is the integer iteration number within $0 \leq n<\infty$. Regarding the microstrip segments that form the Koch dipole, it could be mentioned that they were made of copper on a bakelite substrate and they were $1.5 \mathrm{~mm}$ wide and $4.4 \mathrm{~mm}$ long. Also, it is important to note that, with that segment width, reaching iteration $n=3$ would hinder the construction of the PCB antenna because the length of such segments would be $1.1 \mathrm{~mm}$ (shorter than the width), which is why iteration $n=2$ was chosen. The fractal antenna is matched to a $50 \Omega$ SMA coaxial connector that in turn is connected to a $50 \Omega$ UHF transceiver through a $50 \Omega$ coaxial cable. Figure 1 shows (a) iterations $n=0, n=1$, and $n=2$ for a square Koch curve generated using Wolfram Demonstrations Project available in [19], (b) the top side of the printed fractal antenna, and (c) the bottom side of the printed fractal antenna.

2.2. Simulation. In order to computationally evaluate the performance of the antenna design, it was decided to simulate it using the finite element method considering for the model two subdomains: one to simulate far-field free-space conditions which in turn was subdivided into two regions and the other one to simulate the $2 \mathrm{~mm}$ thick bakelite substrate. Also, an infinite sphere of radiation was considered. On the substrate, three negligible-thickness sheets were considered: two of them for the antenna arms set as finite conductivity and the other one for a $50 \Omega$ lumped port which simulates the antenna feed. A frequency sweep considering 101 points from 400 to $500 \mathrm{MHz}$ was performed in order to evaluate frequency-dependent parameters as reflection coefficient $S_{11}$, standing wave ratio VSWR, and input impedance which are defined as follows [12]:

$$
\begin{array}{r}
S_{11}=\frac{Z_{A}-Z_{0}}{Z_{A}+Z_{0}}, \\
\operatorname{VSWR}=\frac{1-\left|S_{11}\right|}{1+\left|S_{11}\right|},
\end{array}
$$

where $Z_{A}=R_{A}+j X_{A}$ is the input impedance of the antenna and $Z_{0}$ is the impedance to which the designed antenna was connected. Also, an individual analysis setup at $436.5 \mathrm{MHz}$ 


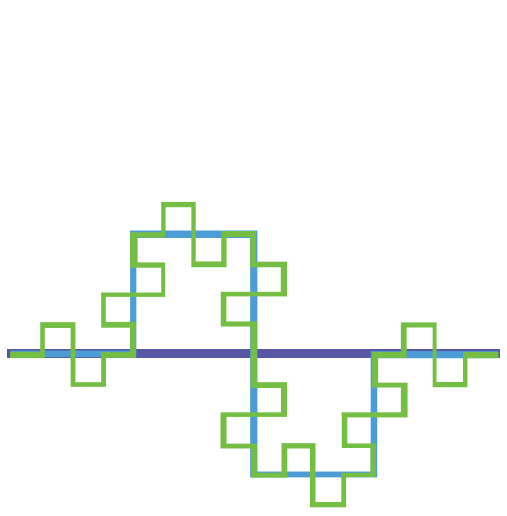

(a)

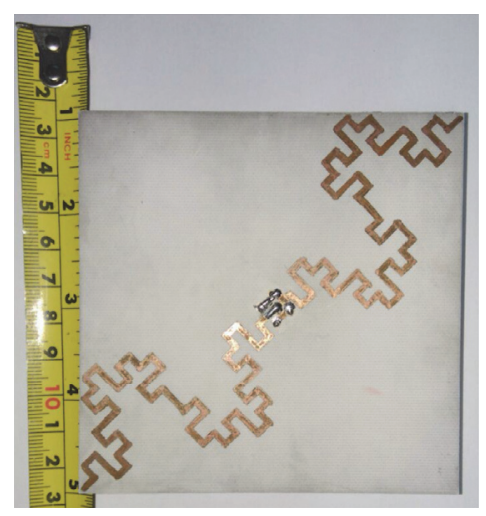

(b)

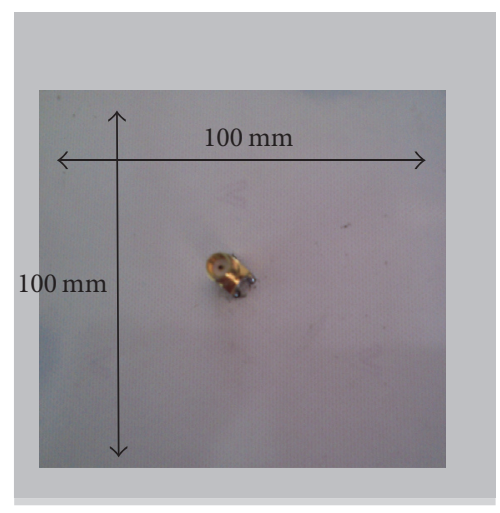

(c)

FIGURE 1: (a) Iterations $n=0, n=1$, and $n=2$ for a square fractal curve used as dipole arms; (b) top side of the PCB for the designed antenna; (c) bottom side of the printed fractal antenna.

was considered to know the gain radiation patterns which are defined by

$$
\begin{aligned}
G_{r e}(\theta, \phi) & =e_{r} G(\theta, \phi)=e_{r} e_{c d} D(\theta, \phi) \\
& =\left[1-\left|S_{11}\right|^{2}\right] e_{c d}\left[4 \pi \frac{U(\theta, \phi)}{P_{\mathrm{rad}}}\right],
\end{aligned}
$$

where $G(\theta, \phi)$ is the antenna gain and $G_{r e}(\theta, \phi)$ is called the realized gain that takes into account the reflection/mismatch losses due to the connection of the antenna element to the transmission line. If the antenna is matched to the transmission line, that is, the antenna input impedance $Z_{A}$ is equal to the characteristic impedance $Z_{0}$ of the transmission line $\left(\left|S_{11}\right|=0\right)$, then the two gains are equal $\left(G_{r e}=\right.$ $G)$. Likewise, $e_{r}$ and $e_{c d}$ are the reflection and radiation efficiencies, respectively (both dimensionless), $D(\theta, \phi)$ is the directivity (dimensionless), $U(\theta, \phi)$ is the radiation intensity, and $P_{\mathrm{rad}}$ is the radiated power [12]. Radiation patterns were simulated at $436.5 \mathrm{MHz}$ because that is the center frequency at which the transceiver used for the satellite implementation described in Section 2.4 works.

2.3. Antenna Fabrication. The fabrication step was conducted by using conventional PCB techniques. For the design of the antenna geometry, EDA (electronic design automation) software for the creation of professional schematics and printed circuit boards was used [20]. This fabrication process included an SMA (SubMiniature version A) connector, which was connected directly to the antenna arms. As it was mentioned before, the microstrip Koch fractal antenna was built on the total diagonal of a $100 \mathrm{~mm} \times 100 \mathrm{~mm}$ single-sided commercial PCB card shown in Figure 1(b).

2.4. Measurements and Implementation. Measurements in this study were carried out in two stages. The first stage was in a conventional laboratory for measuring frequencydependent parameters as $S_{11}$, VSWR, and input impedance from 400 to $500 \mathrm{MHz}$. This first stage was conducted using an N5222A vector network analyzer (VNA) [21], for which 1-port SOLT (short-open-load-thru) calibration based on an $85521 \mathrm{~A} 3.5 \mathrm{~mm}$ calibration kit was performed [22]. Data produced by the VNA were exported as .csv file for plotting and comparison with simulation results, where the same 101 frequencies were considered. The second stage where radiation patterns were measured was a professional anechoic chamber, which meets the international standards MIL-STD 461E, CISPR 25, RTCA DO 160, ISO 11452-2/SAE J111321, CISPR 12, and NSA (CISPR 16/ANSI C63.4). It has free dimensions of $12 \mathrm{~m} \times 8 \mathrm{~m} \times 6 \mathrm{~m}$ and includes EMC$24 \mathrm{PCL}^{\circledR}$ high performance pyramidal absorbing material on its inner walls [23]. The experimental setup for measuring radiation patterns consisted of the designed fractal antenna placed vertically, which in turn was powered by a $-20 \mathrm{dBm}$ RF generator at $436.5 \mathrm{MHz}$, the same frequency used for simulated radiation patterns. The fractal antenna was located $3 \mathrm{~m}$ separated from a log-periodic receiving antenna to ensure far-field conditions. Both the transmitting antenna and the receiving antenna were $2.5 \mathrm{~m}$ above the ground, while the transmitting antenna (fractal) was turning on its axis. Figure 2 shows (a) the experimental setup using the anechoic chamber and (b) the inside of the anechoic chamber.

Finally, in order to test the operation of the designed antenna for picosatellite applications, it was decided to implement a communication link between the small satellite and a ground station working at the 70-centimeter band. The link was carried out by placing the ground station on a roof, where a line of sight with the top of a hill at the edge of the city is presented. The approximate coordinates of the ground station were $22.7686638,-102.5639583$ at $2418 \mathrm{~m}$ above sea level, while for the picosatellite (the top of the hill) they were $22.7769250,-102.5638166$ at $2605 \mathrm{~m}$ above sea level. Since the ground station and the picosatellite are relatively close, the curvature of the earth is neglected and therefore a line-of-sight distance of $938.81 \mathrm{~m}$ with an angle to the horizontal of $11.49^{\circ}$ was computed. The ground station consisted of a UHF vertical monopole antenna connected to an RF receiver card that in turn was connected to a laptop computer. The satellite through the designed fractal antenna sent telemetry data to the ground station at $436.675 \mathrm{MHz}$, one 


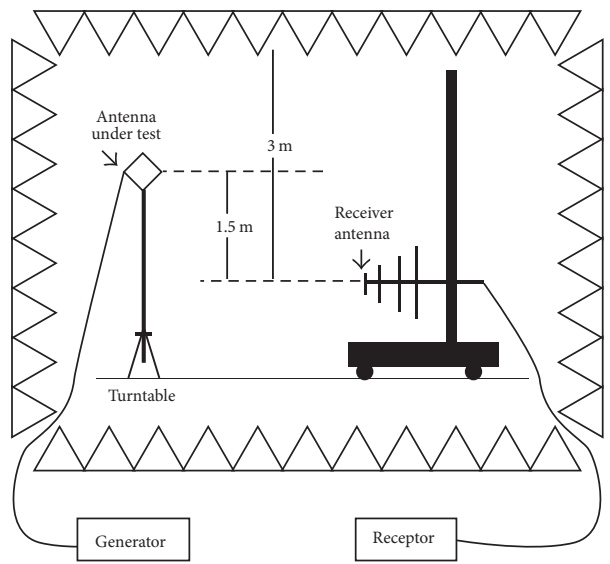

(a)

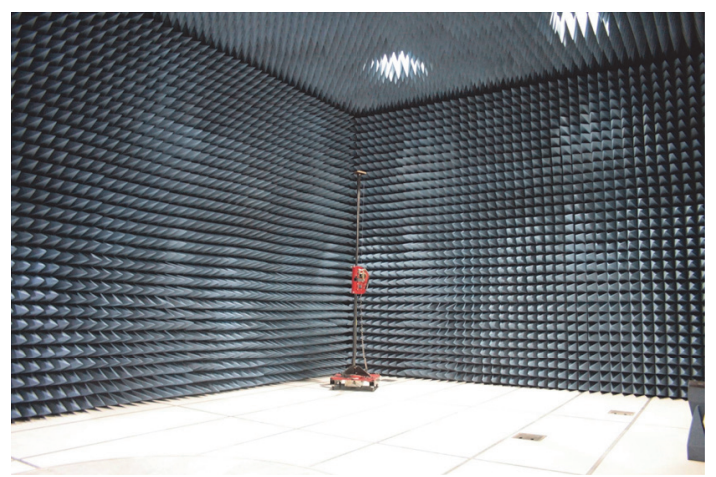

(b)

Figure 2: Experimental setup using an anechoic chamber: (a) diagram and (b) a photo of the inside of the chamber.

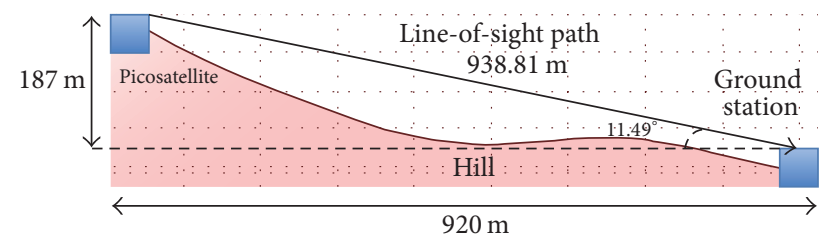

Figure 3: The diagram of the prototype satellite link implemented.

of the frequency channels at which the transceiver TR2M436.50-10-ARS from Radiometrix ${ }^{\circledR}$ works. TR2M-436.50-10ARS was used to build the communications stage for the small satellite and the receiving card for the ground station. Polarization of transmitting and receiving antennas was set to be matched so that losses due to polarization mismatch were minimized. Figure 3 shows the diagram of the prototype satellite link implemented. It is important to highlight that, in this scenario, there were no surrounding structures and multipaths occur due to ground reflections. However, due to the distance between the transmitter and the receiver and because a narrowband signal was transmitted, the multipath waves could be neglected.

\section{Results and Discussion}

Regarding frequency-dependent parameters, such as reflection coefficient $S_{11}$, VSWR, and input antenna impedance in the range of 400 to $500 \mathrm{MHz}$ (70-centimeter band), it can be highlighted that simulation results were very similar to those obtained in measurements with the VNA, in both cases observing resonance frequencies with reflection coefficients below $-20 \mathrm{~dB}$, VWSR under 2, and impedance with magnitude very close to $50 \mathrm{ohms}$. Figure 4 shows the antenna behavior in terms of (a) reflection coefficient, (b) VSWR, and (c) input antenna impedance.

In order to highlight the results observed in the above graphics, emphasis on the resonance frequencies and their bandwidths around them was done, so in Table 1 a summary of the antenna performance in terms of frequency-dependent parameters is shown.

As it was mentioned in Sections 2.2 and 2.4, two frequencies of interest in this work are $436.5 \mathrm{MHz}$ and $436.675 \mathrm{MHz}$ : the central frequency of transceiver TR2M-436.50-10-ARS and the frequency channel selected for the practical satellite implementation, respectively. Although these are not optimum frequencies for the fractal antenna, frequencydependent parameters remain within acceptable limits. Table 2 shows the fractal antenna behavior at $436.5 \mathrm{MHz}$ and $436.675 \mathrm{MHz}$.

In this work, it was decided to design this linearly polarized fractal antenna on the diagonal of one side of a CubeSat in order to make the most of its limited dimensions compared with wavelengths at 70-centimeter band. Although the fractal antenna does not have its resonance frequency within the TR2M-436.50-10-ARS band, the performance is acceptable without designing one that resonates exactly at those frequencies, a design where the main constraint is the total length that would be greater retaining the same iteration.

As it was mentioned before, regarding radiation patterns for the printed fractal antenna at $436.5 \mathrm{MHz}$, they were obtained both by simulations and by measurements. The fractal antenna was placed vertically so that the PCB formed a diamond with respect to the floor, considering the PCB plane as the " $x y$ " plane with the " $z$-axis" perpendicular to this plane. Figure 5 shows (a) the 3D simulated total gain pattern with respect to the PCB geometry and the corresponding magnitude of the current distribution on the antenna surface, (b) plots for 2D simulated normalized total gain pattern for the main antenna planes, and (c) plots for $2 \mathrm{D}$ measured normalized patterns (power at the receiver) for the main antenna planes inside an anechoic chamber.

In Figure 5, it can be seen that both the simulated and the measured radiation patterns show similar behaviors, although it should be mentioned that simulation case was ideal, while measurements were performed using different antennas at the transmitter and at the receiver. Anyway, in both cases, the typical behavior of dipole antennas is observed, which leads to classifying it as an omnidirectional 
TABLE 1: Comparison between simulations and measurements for the designed fractal antenna.

\begin{tabular}{lccccc}
\hline & $S_{11}(\mathrm{~dB})$ & $-10 \mathrm{~dB}$ bandwidth $(\mathrm{MHz})$ & VSWR & Input impedance $(\Omega)$ & Magnitude of input impedance $(\Omega)$ \\
\hline Simulated resonance at $462 \mathrm{MHz}$ & -25.62 & 43 & 1.111 & $55.41-\mathrm{j} 1.102$ & 55.42 \\
Measured resonance at $455 \mathrm{MHz}$ & -22.83 & 50 & 1.156 & $55.71-\mathrm{j} 5.077$ & 56 \\
\hline
\end{tabular}

TABLE 2: Frequency-dependent parameters for the designed Koch antenna at $436.5 \mathrm{MHz}$ and $436.675 \mathrm{MHz}$.

\begin{tabular}{lcccc}
\hline & $S_{11}(\mathrm{~dB})$ & VSWR & Input impedance $(\Omega)$ & Magnitude of input impedance $(\Omega)$ \\
\hline Simulations at $436.5 \mathrm{MHz}$ & -8.486 & 2.208 & $49.59-\mathrm{j} 40.475$ & 64.01 \\
Measurements at $436.5 \mathrm{MHz}$ & -11.99 & 1.6725 & $40.605-\mathrm{j} 21.45$ & 45.82 \\
Simulations at $436.675 \mathrm{MHz}$ & -8.54 & 2.1965 & $49.49-\mathrm{j} 41.23$ & 64.41 \\
Measurements at $436.675 \mathrm{MHz}$ & -11.74 & 1.6636 & $40.72-\mathrm{j} 21.27$ & 45.94 \\
\hline
\end{tabular}

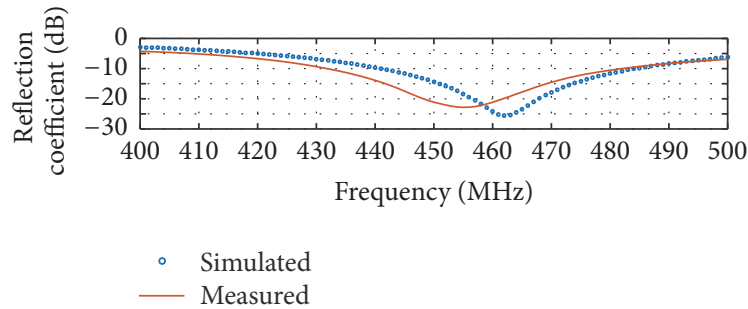

(a)

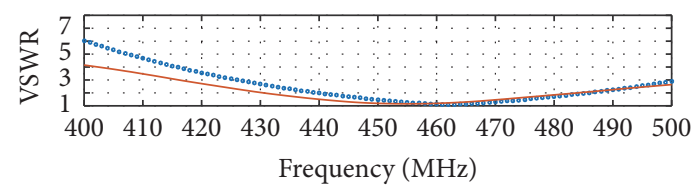

- Simulated

- Measured

(b)

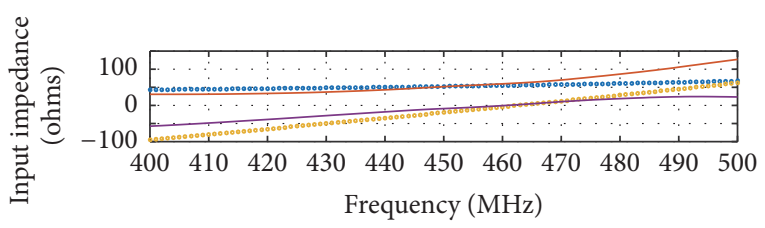

- Simulated resistance $\quad$ Simulated reactance

— Measured resistance - Measured reactance

(c)

FIGURE 4: Frequency-dependent parameters describing the antenna behavior: (a) reflection coefficient, (b) VSWR, and (c) input impedance from 400 to $500 \mathrm{MHz}$.

antenna [12]. At $436.5 \mathrm{MHz}$, simulated and measured radiation patterns have nulls on the E-plane at $90^{\circ}$ and $270^{\circ}$ while they have main lobes at $0^{\circ}$ and $180^{\circ}$. At $436.5 \mathrm{MHz}$, the maximum total gain computed by numerical simulations was $G_{\max }=1.391=1.433 \mathrm{dBi}$, while at $462 \mathrm{MHz}$ it was $G_{\max }=1.433=1.565 \mathrm{dBi}$ which is the simulated resonance frequency between $400 \mathrm{MHz}$ and $500 \mathrm{MHz}$. Simulated radiation efficiencies were $98.56 \%$ and $98.77 \%$ at $436.5 \mathrm{MHz}$ and $462 \mathrm{MHz}$, respectively. An aspect to emphasize with respect to the small satellites is that if they lack an on-board stabilization system, this results in a polarization mismatch between the involved antennas. Figure 6 shows simulated components (a) $G_{\theta}(\theta)$ and (b) $G_{\emptyset}(\theta)$ of the antenna gain at $462 \mathrm{MHz}$ for different values of $\emptyset$ from $0^{\circ}$ to $180^{\circ}$ where the different radiation patterns can be appreciated and in order to see polarization discrimination.

Finally, with regard to the implementation of the prototype satellite link working at $436.675 \mathrm{MHz}$, telemetry data from the picosatellite including temperature, altitude, and acceleration were received successfully at the ground station. Similarly, it was decided to take measurements of received power with an N9913A spectrum analyzer [24], where it was observed that the received power was $P_{R}=-57.526085 \mathrm{dBm}$ $=1.7676 \mathrm{nW}$. This received power level is acceptable considering that losses due to connectors, adapters, and cables used during this implementation exceed $5 \mathrm{~dB}$. Also, a receiving antenna gain of approximately $5.56 \mathrm{dBi}$, separation between transmitter and receiver of $938.81 \mathrm{~m}$, and transmitted power of $27.31 \mathrm{dBm}=538.27 \mathrm{~mW}$ were considered which led to the estimation of a transmitting antenna gain that resembles the one computed by simulations using the Friis equation under ideal conditions [25]. Figure 7 shows (a) the antenna design connected to the picosatellite, (b) the ground station with its UHF vertical monopole antenna getting telemetry data, and (c) the UHF vertical monopole antenna connected to the spectrum analyzer.

\section{Conclusion}

In this work, the use of fractal geometries for designing printed antennas at the 70-centimeter band for TT\&C downlink applications in small satellites has been demonstrated. It is probed that an antenna working at 70-centimeter band can be printed on the limited surfaces of CubeSat faces $(100 \mathrm{~mm} \times 100 \mathrm{~mm})$, since, for example, conventional $\lambda / 2$ dipoles working at those frequencies are about $35 \mathrm{~cm}$ long and could not fit within those areas. The design was a second-iteration fractal dipole antenna printed on a $100 \mathrm{~mm}$ $\times 100 \mathrm{~mm}$ commercial PCB card with bakelite substrate and having a total length including the feed gap of $56.56 \mathrm{~cm}$. Frequency-dependent parameters such as reflection coefficient $S_{11}$, voltage standing wave ratio VSWR, and impedance magnitude $\left|Z_{A}\right|$ were obtained, with resonance frequencies appearing at $462 \mathrm{MHz}$ and $455 \mathrm{MHz}$ for simulations and measurements, respectively, at which $S_{11}<-20 \mathrm{~dB}$, VSWR 

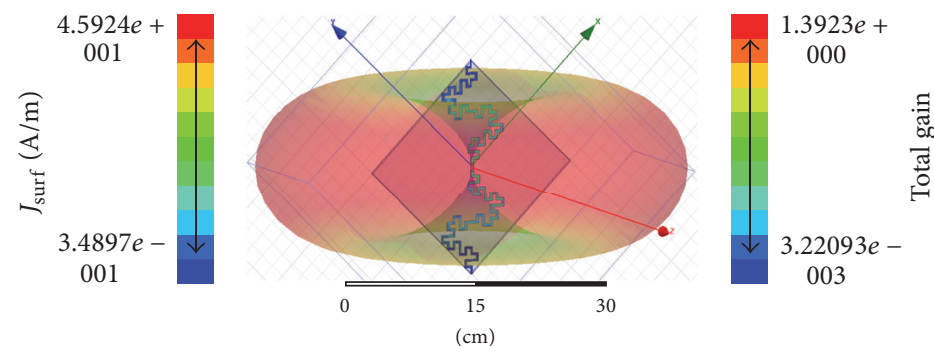

(a)

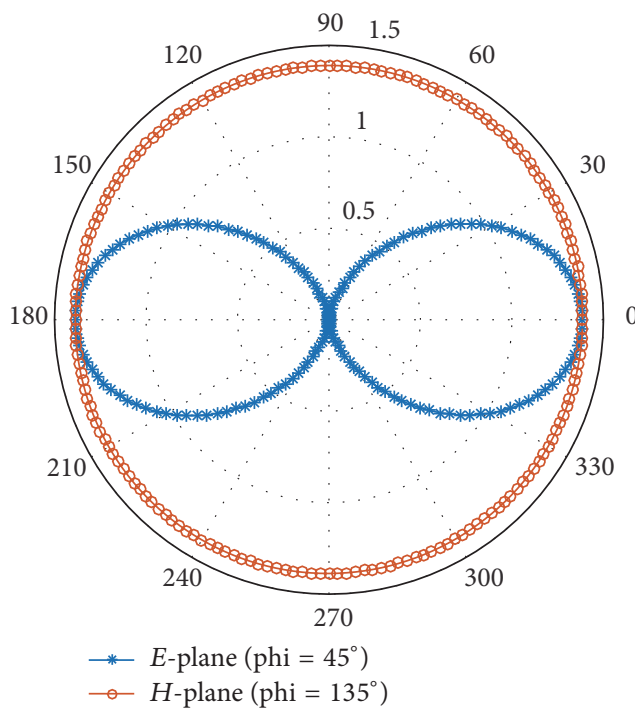

(b)

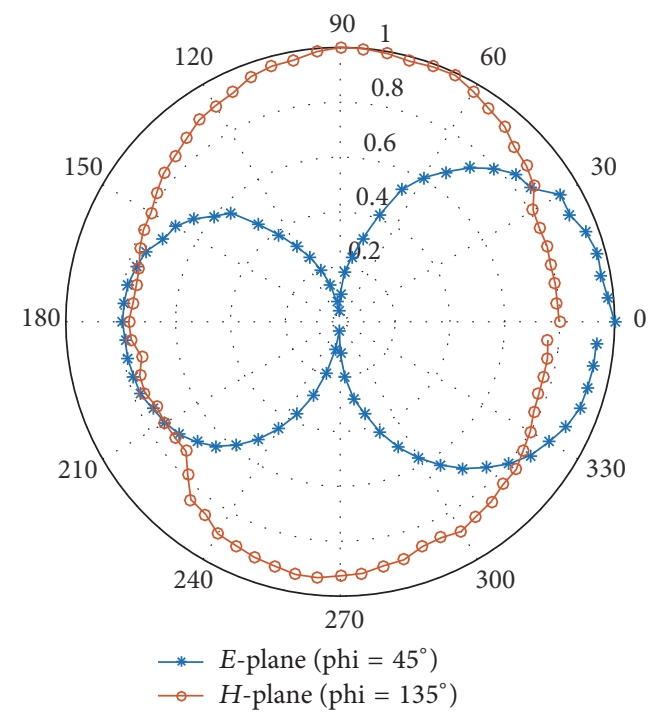

(c)

Figure 5: Radiation patterns at $436.5 \mathrm{MHz}$ : (a) 3D simulated total gain pattern with respect to the PCB geometry and its corresponding magnitude of the current distribution on the antenna surface, (b) 2D plots for simulated normalized total gain patterns for the main antenna planes, and (c) 2D plots for measured normalized power patterns for the main antenna planes inside an anechoic chamber.

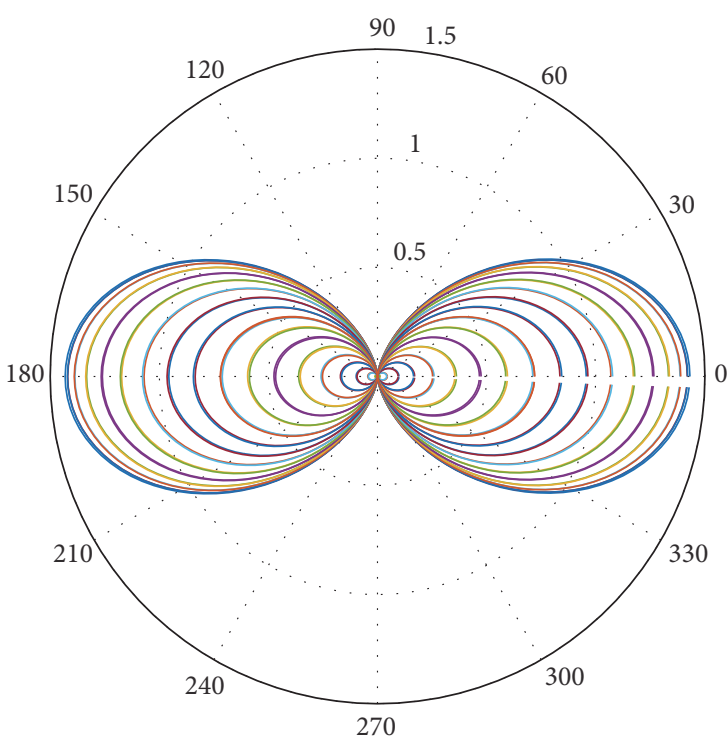

(a)

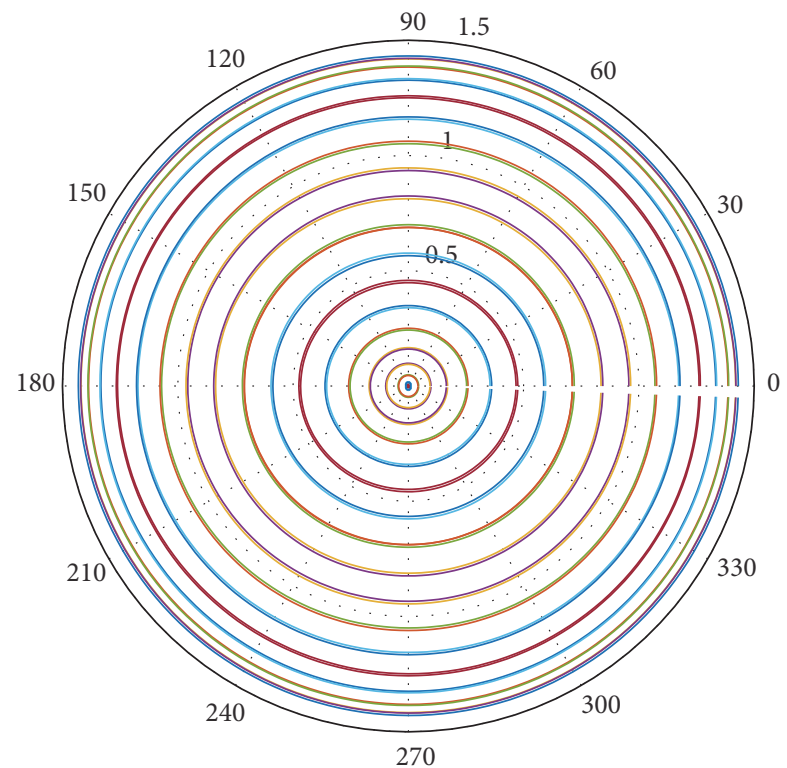

(b)

Figure 6: Simulated components (a) $G_{\theta}(\theta)$ and (b) $G_{\emptyset}(\theta)$ of the antenna gain at $462 \mathrm{MHz}$ for different values of $\emptyset$ from $0^{\circ}$ to $180^{\circ}$. 


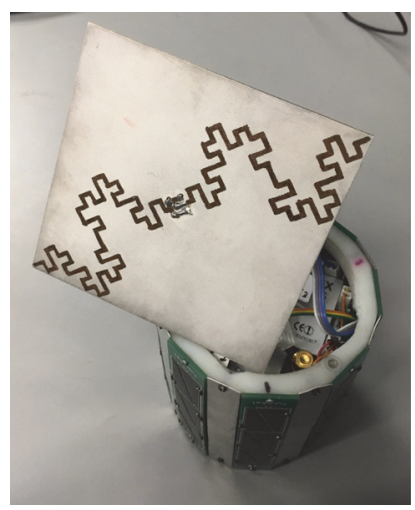

(a)

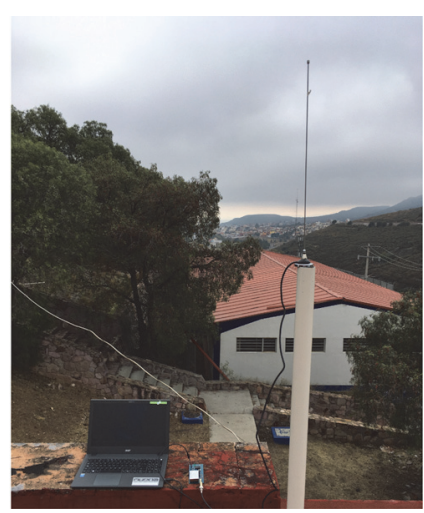

(b)

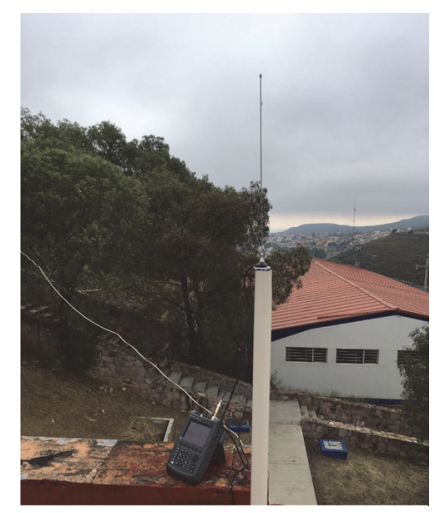

(c)

Figure 7: (a) The antenna design connected to the picosatellite, (b) the ground station with its UHF vertical monopole antenna getting telemetry data, and (c) the UHF vertical monopole antenna connected to the spectrum analyzer.

$<1.2$, and $\left|Z_{A}\right| \approx 50 \Omega$. Radiation patterns at $436.5 \mathrm{MHz}$ were simulated and measured using a professional anechoic chamber. A simulated maximum antenna gain of 1.433 at the simulation resonance frequency was obtained. Finally, a satellite prototype implementation at $436.675 \mathrm{MHz}$ was carried out in order to test carrier signal reception and transmission of telemetry signals using the antenna design, where an approximate link budget considering losses gives a maximum gain similar to that obtained through simulations. These parameters make this antenna geometry suitable for TTC subsystems in CubeSat satellites, which leaves as future work the experimentation with other types of substrates in order to improve the antenna characteristics.

\section{Competing Interests}

The authors declare that there are no competing interests regarding the publication of this paper.

\section{Acknowledgments}

This work was supported by Project 3066 of "Centro de Investigación y Desarrollo en Telecomunicaciones Espaciales" from "Cátedras CONACYT" program, Project 241947 of "Universidad de Colima” from "Fondo Mixto CONACYTGobierno del Estado de Colima," and Project 248410 of "Centro de Investigación y Desarrollo en Telecomunicaciones Espaciales" from "Fondo Sectorial CONACYT-AEM." The authors thank the technical staff at "Laboratorio de Radiofrecuencia," Universidad de Colima, for their support and collaboration and Dr. Israel Alejandro Arriaga Trejo, CONACYT researcher at the Autonomous University of Zacatecas, for the feedback provided to this work.

\section{References}

[1] http://www.nasa.gov/audience/forstudents/5-8/features/nasaknows/what-is-a-satellite-58.html.

[2] B. R. Elbert, The Satellite Communications Applications Handbook, Artech House Space Applications Series, 2nd edition, 2004.
[3] I. Marboe, Small Satellites: Regulatory Challenges and Chances, Studies in Space Law, 2016.

[4] C. Kakoyiannis and P. Constantinou, "Electrically small microstrip antennas targeting miniaturized satellites: the CubeSat paradigm," in Microstrip Antennas, N. Nasimuddin, Ed., chapter 12, InTech, Rijeka, Croatia, 2011.

[5] S. Gao, M. Brenchley, M. Unwin et al., "Antennas for small satellites," in Proceedings of the Loughborough Antennas and Propagation Conference (LAPC '08), pp. 66-69, Loughborough, UK, March 2008.

[6] S. Gao, K. Clark, M. Unwin et al., "Antennas for modern small satellites," IEEE Antennas and Propagation Magazine, vol. 51, no. 4, pp. 40-56, 2009.

[7] A. K. A. N. Volkan, "Electrically small printed antenna for applications on cubesat and nano-satellite platforms," Microwave and Optical Technology Letters, vol. 57, no. 4, pp. 891-896, 2015.

[8] K. Clark, M. Unwin, J. Zackrisson et al., "Microstrip antenna with solar cells for microsatellites," in Proceedings of the Antennas and Propagation Society International Symposium Digest, vol. 2, pp. 786-789, Seattle, Wash, USA, June 1994.

[9] R. Mathur, R. Haupt, and C. Swenson, "Student antenna design for a nanosatellite," in Proceedings of the IEEE Aerospace Conference, vol. 7, pp. 3683-3688, Big Sky, Mont, USA, March 2001.

[10] K. Muchalski, M. Jagoda, M. Tomasiak et al., "Optimizing TT\&C antenna placement on minisatellites," in Proceedings of the 15th International Conference on Microwaves, Radar and Wireless Communications (MIKON '04), pp. 489-492, Warzaw, Poland, May 2004.

[11] T. J. Mizuno, J. D. Roque, B. T. Murakami et al., "Antennas for distributed nanosatellite networks," in Proceedings of the IEEE/ACES International Conference on Wireless Communications and Applied Computational Electromagnetics, pp. 606-609, IEEE, Honolulu, Hawaii, USA, April 2005.

[12] C. A. Balanis, Modern Antenna Handbook, John Wiley \& Sons, 2009.

[13] B. Hephzibah Lincy, A. Srinoivasan, and B. Rajalakshmi, "Wideband fractal microstrip antenna for wireless application," in Proceedings of the International Conference on Human Machine Interaction, Chennai, India, March 2013.

[14] B. B. Mandelbrot, The Fractal Geometry of Nature, W. H. Freeman and Company, New York, NY, USA, 1983. 
[15] Y. Suo, J. Han, W. Li, and W. Deng, "Design and analysis of miniature fractal antenna," in Proceedings of the Progress in Electromagnetics Research Symposium (PIERS '14), pp. 17991803, Guangzhou, China, August 2014.

[16] J. P. Gianvittorio and Y. Rahmat-Samii, "Fractal antennas: a novel antenna miniaturization technique, and applications," IEEE Antennas and Propagation Magazine, vol. 44, no. 1, pp. 20 36, 2002.

[17] C. P. Baliarda, J. Romeu, and A. Cardama, "The Koch monopole: a small fractal antenna," IEEE Transactions on Antennas and Propagation, vol. 48, no. 11, pp. 1773-1781, 2000.

[18] A. Mondal, "Miniaturized and dual band hybrid koch dipole fractal antenna design," International Journal of Computer Communication and Information System, vol. 2, no. 1, pp. 23-27, 2010.

[19] http://demonstrations.wolfram.com/SquareKochFractalCurves/.

[20] J. M. Hughes, Practical Electronics: Components and Techniques, O’Reilly Media Inc, Newton, Mass, USA, 2015.

[21] http://www.keysight.com/en/pdx-x201875-pn-N5222A/pnamicrowave-network-analyzer-265-ghz?pm=spc\&nid= $-32497.1150215 \& c c=M X \& l_{c}=$ spa.

[22] http://www.keysight.com/en/pd-2171664-pn-85521A/4-in-1oslt-mechanical-calibration-kit-dc-to-265-ghz-type-35-mm-f$50-\mathrm{ohm}$ ? cc=US\&lc=eng.

[23] http://www.ets-lindgren.com/AnechoicChamber.

[24] http://www.keysight.com/en/pdx-x201901-pn-N9913A/fieldfox-handheld-microwave-analyzer-4-ghz?cc=US\&lc=eng.

[25] V. L. Granatstein, Physical Principles of Wireless Communications, CRC Press, Taylor \& Francis Group, Boca Raton, Fla, USA, 2nd edition, 2012. 


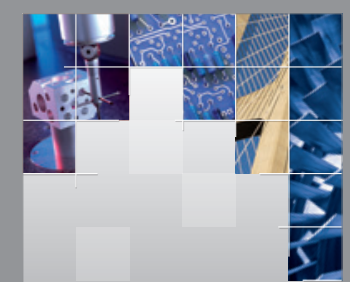

\section{Enfincering}
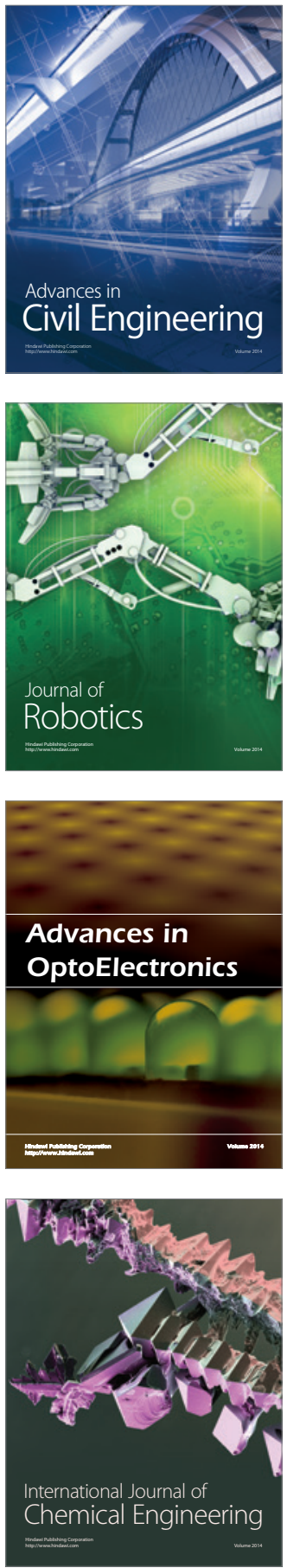

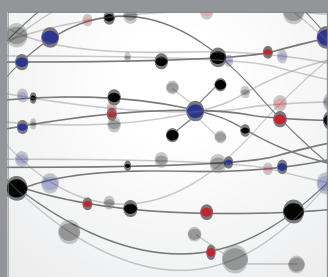

The Scientific World Journal

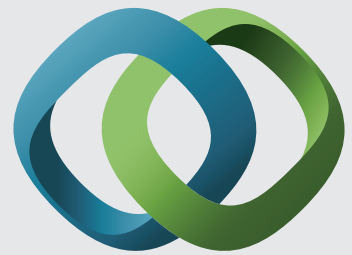

\section{Hindawi}

Submit your manuscripts at

https://www.hindawi.com
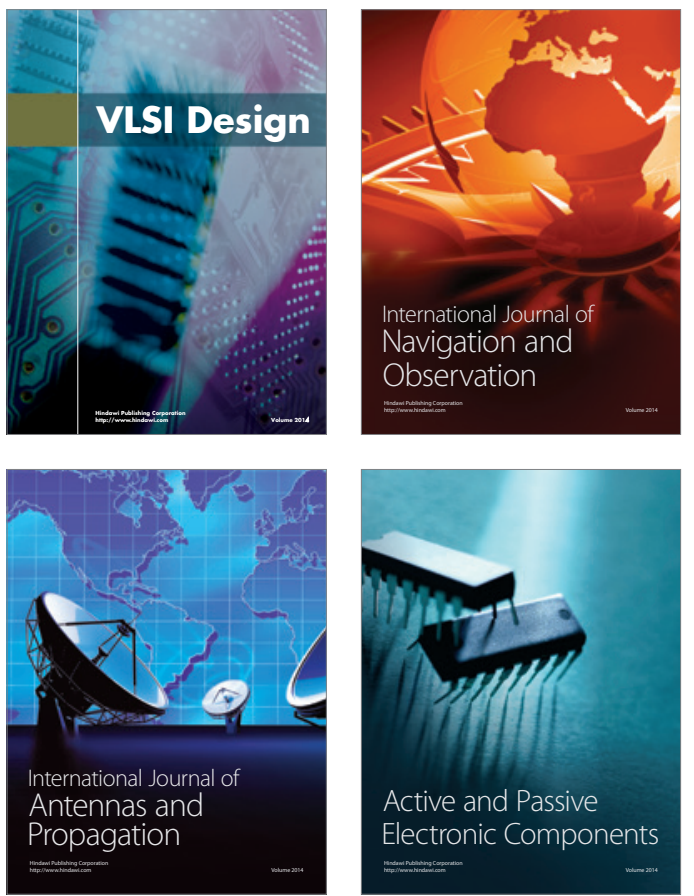
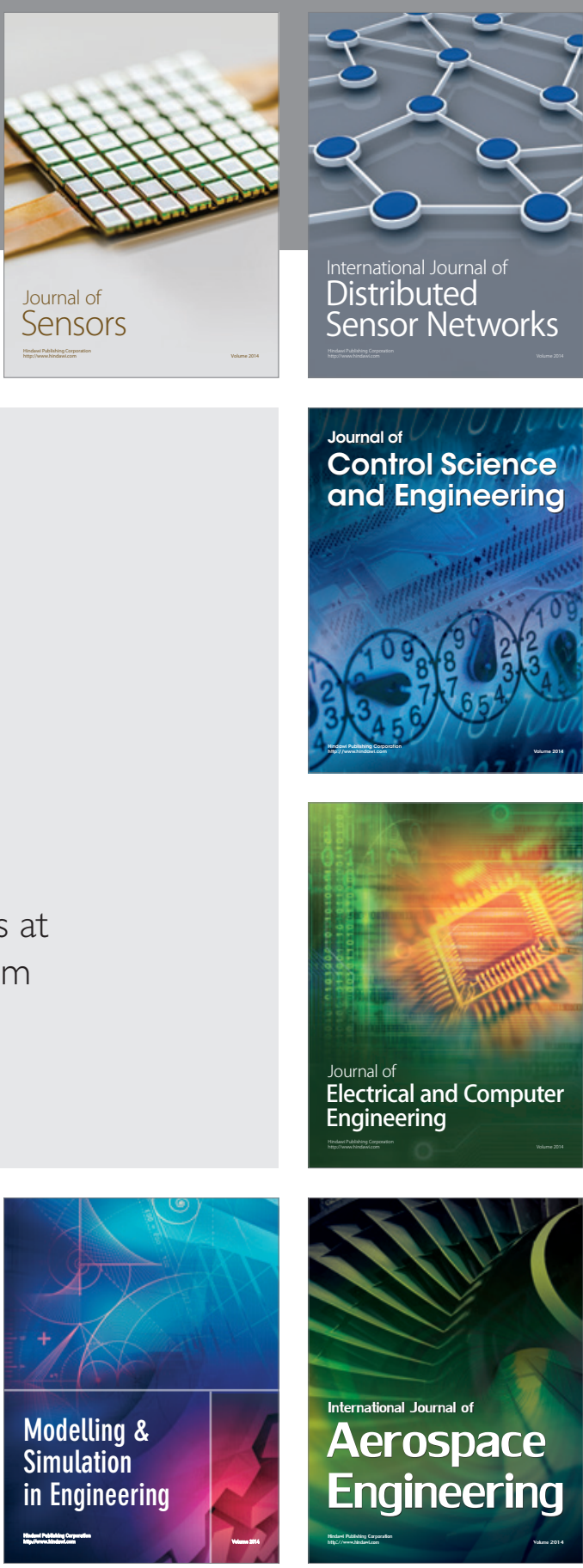

International Journal of

Distributed

Sensor Networks

$-$

Joumal of

Control Science

and Engineering
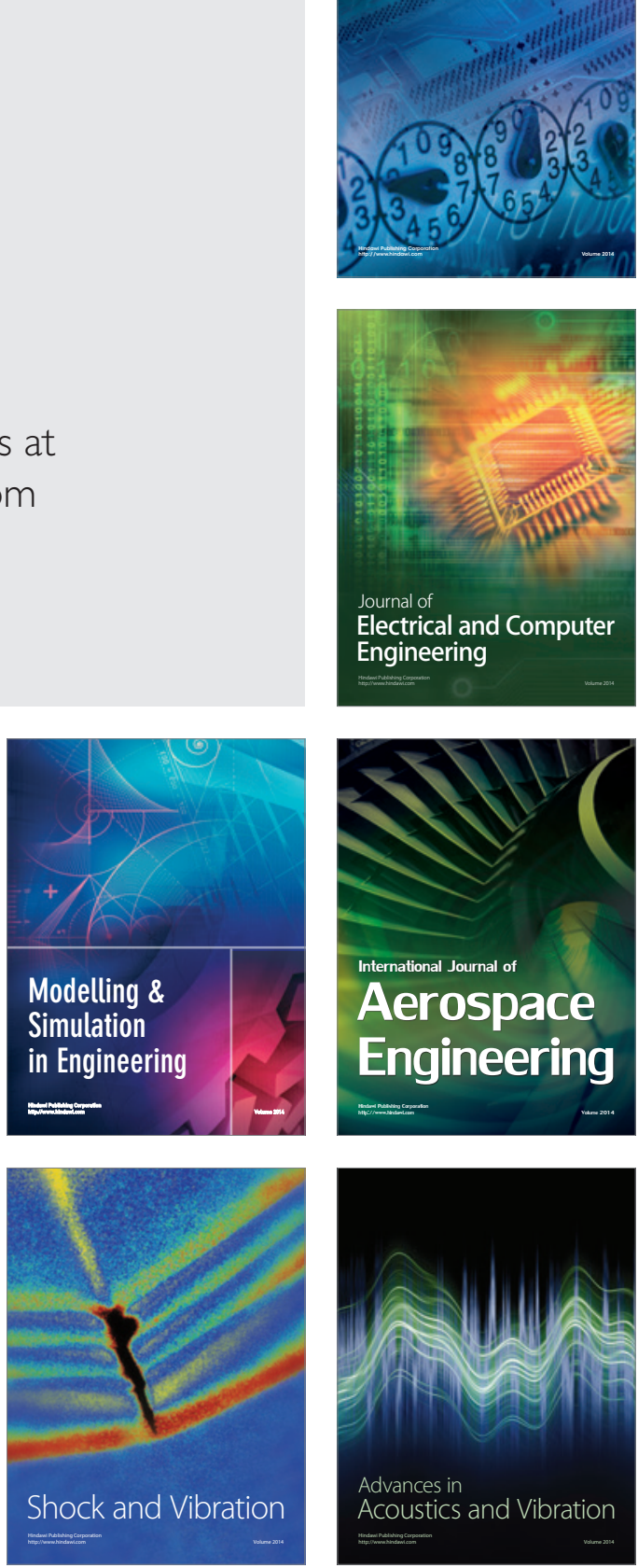